1. Djafarzadeh, R., et al. 2000. Biophysical and structural characterization of proton-translocating NADH-dehydrogenase (complex I) from the strictly aerobic yeast Yarrowia lipolytica. Biochim. Biophys. Acta. 1459:230-238.

2. Hirst, J. 2005. Energy transduction by respiratory complex I-an evaluation of current knowledge. Biochem. Soc. Trans. 33:525-529.

3. Carroll, J., Fearnley, I.M., Shannon, R.J., Hirst, J., and Walker, J.E. 2003. Analysis of the subunit composition of complex I from bovine heart mitochondria. Mol. Cell Proteomics. 2:117-126.

4. Grigorieff, N. 1998. Three-dimensional structure of bovine NADH:ubiquinone oxidoreductase (complex I) at $22 \mathrm{~A}$ in ice. J. Mol. Biol. 277:1033-1046.

5. Antonicka, H., et al. 2003. Identification and characterization of a common set of complex I assembly intermediates in mitochondria from patients with complex I deficiency. J. Biol. Chem. 278:43081-43088.

6. Ugalde, C., et al. 2004. Human mitochondrial complex I assembles through the combination of evolutionary conserved modules: a framework to interpret complex I deficiencies. Hum. Mol. Genet. 13:2461-2472.

7. Schulte, U. 2001. Biogenesis of respiratory complex I. J. Bioenerg. Biomembr. 33:205-212.

8. Brandt, U., et al. 2005. Structure-function relationships in mitochondrial complex I of the strictly aerobic yeast Yarrowia lipolytica. Biochem. Soc. Trans. 33:840-844.

9. Triepels, R.H., Van Den Heuvel, L.P., Trijbels, J.M., and Smeitink, J.A. 2001. Respiratory chain complex I deficiency. Am. J. Med. Genet. 106:37-45.

10. von Kleist-Retzow, J.C., et al. 1998. A high rate (20\%$30 \%$ ) of parental consanguinity in cytochrome-oxidase deficiency. Am. J. Hum. Genet. 63:428-435.

11. OMIM - Online Mendelian Inheritance in Man. http://www.ncbi.nlm.nih.gov/entrez/query. fcgi? db=OMIM.

12. Robinson, B.H. 1998. Human complex I deficiency: clinical spectrum and involvement of oxygen free radicals in the pathogenicity of the defect. Biochim. Biophys. Acta. 1364:271-286.

13. Benit, P., et al. 2004. Mutant NDUFS3 subunit of mitochondrial complex I causes Leigh syndrome. J. Med. Genet. 41:14-17.

14. Rotig, A., et al. 2004. Molecular diagnostics of mitochondrial disorders. Biochim. Biophys. Acta. 1659:129-135

15. de Lonlay, P., et al. 2001. A mutant mitochondrial respiratory chain assembly protein causes complex III deficiency in patients with tubulopathy, encephalopathy and liver failure. Nat. Genet. 29:57-60.

16. Janssen, R., Smeitink, J., Smeets, R., and van Den Heuvel, L. 2002. CIA30 complex I assembly factor: a candidate for human complex I deficiency? Hum. Genet. 110:264-270.

17. Ogilvie, I., Kennaway, N.G., and Shoubridge, E.A. 2005. A molecular chaperone for mitochondrial complex I assembly is mutated in a progressive encephalopathy. J. Clin. Invest. 115:2784-2792. doi:10.1172/JCI26020.

18. Smeitink, J., et al. 1998. Molecular characterization and mutational analysis of the human B17 subunit of the mitochondrial respiratory chain complex I. Hum. Genet. 103:245-250.

\title{
PDGF signaling in pulmonary arterial hypertension
}

\author{
Robyn J. Barst
}

New York Presbyterian Pulmonary Hypertension Center, Columbia University College of Physicians \& Surgeons, New York, New York, USA.

\begin{abstract}
The pathobiology of pulmonary arterial hypertension (PAH) includes endothelial cell dysfunction and proliferation and migration of VSMCs. As PDGF has been implicated in these processes, Schermuly et al. hypothesized that altered PDGF signaling may be involved in the vascular remodeling observed in PAH. To explore this notion further, the authors evaluated the effects of the PDGF receptor inhibitor STI571 in 2 different animal models of pulmonary hypertension (see the related article beginning on page 2811). In both models, after development of pulmonary vascular disease, administration of STI571 reversed pulmonary vascular changes. These studies provide preclinical proof of concept for the clinical development of a PDGF inhibitor as a targeted therapy for PAH patients.
\end{abstract}

Pulmonary arterial hypertension (PAH), a disorder limited to the pulmonary circulation, is characterized by pulmonary vascular obstruction and variable pulmonary vasoconstriction leading to increased pulmonary vascular resistance and death. PAH can be idiopathic, or unexplained (formerly termed primary pulmonary hypertension); PAH can also occur in association with connective tissue diseases, HIV infection, congenital heart disease, portal hypertension, and appetite suppressant exposure. Idiopathic PAH occurs more often in women than in men,

Nonstandard abbreviations used: BMP, bone morphogenetic protein; $\mathrm{PAH}$, pulmonary arterial hypertension.

Conflict of interest: The author serves as a consultant to Actelion Ltd., CoTherix Inc., Encysive Pharmaceuticals Inc., INO Therapeutics Inc., Mondo Developments, Myogen Inc., GlaxoSmithKline Inc., Pfizer Inc., and United Therapeutics Corp.

Citation for this article: J. Clin. Invest. 115:2691-2694 (2005). doi:10.1172/JCI26593. with a median survival of 2.8 years if untreated. The mean age at diagnosis is 35 years, i.e., it occurs most often in young adults with no other comorbid conditions. Although current treatment options have markedly improved overall quality of life and survival in PAH, 5-year survival remains at 50\% for this devastating disease. As discussed below, over the past 2 decades, we have learned a great deal about the pathobiology of PAH; however, we still do not know what initiates this disease with its subsequent progressive pulmonary vascular obstruction.

PAH has a multifactorial pathobiology. Vasoconstriction, remodeling of the pulmonary vessel wall, and thrombosis contribute to increased pulmonary vascular resistance in PAH. The process of pulmonary vascular remodeling involves all layers of the vessel wall and is complicated by cellular heterogeneity within each compartment of the pulmonary arterial wall (Fig- ure 1). Indeed, each cell type (endothelial, smooth muscle, and fibroblast), as well as inflammatory cells and platelets, may play a significant role in PAH. Endothelial dysfunction is considered a key element in the pathobiology of $\mathrm{PAH}$, with increased levels of endothelin occurring concomitantly with decreased $\mathrm{NO}$ and prostacyclin levels (1). Although it remains unclear whether excessive vasoconstriction is associated with the endothelial dysfunction, many of the perturbations associated with the endothelial dysfunction promote vascular remodeling in addition to increasing pulmonary vascular tone. Prostacyclin, $\mathrm{NO}$, endothelin, angiopoietin I, serotonin, cytokines, chemokines, and members of the TGF- $\beta$ superfamily have all been implicated in the pathobiology of PAH. As a result, 3 of the currently approved therapeutic modalities for the treatment of $\mathrm{PAH}$ - prostacyclins, phosphodiesterase inhibitors, and endothelin receptor antagonists (2-4) - target prostacyclin and NO deficiencies and increased endothelin levels, respectively, in $\mathrm{PAH}$ patients.

Distal extension of smooth muscle cells into small, peripheral, normally nonmuscular pulmonary arterioles is a hallmark of PAH. However, the processes causing this migration as well as the formation of a layer of microfibroblasts and extracellular matrix (termed the neointima) between the endothelial cells and the internal elas- 


\section{Pulmonary vascular remodeling}

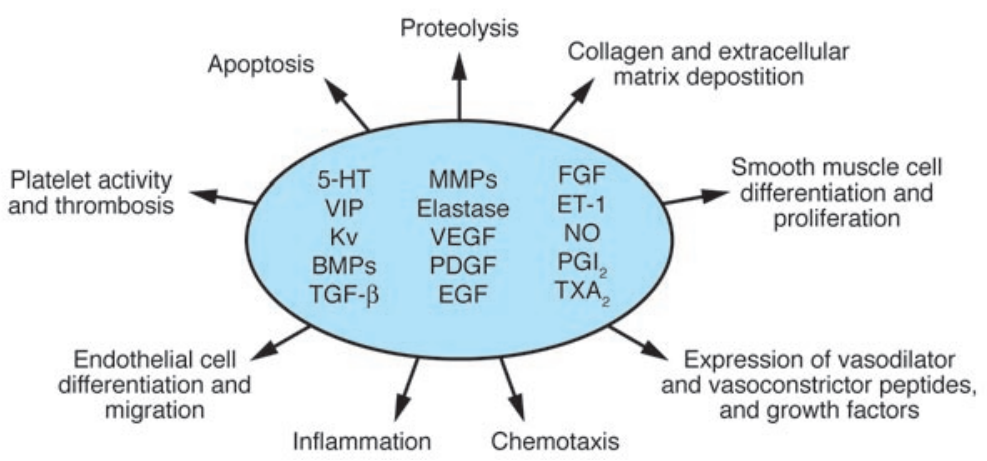

Figure 1

Cellular and molecular pathobiology of pulmonary vascular remodeling in PAH. ET-1, endothelin 1; 5-HT, serotonin; Kv, potassium channel; $P \mathrm{Fl}_{2}$, prostacyclin; $T X A_{2}$, thromboxane $A_{2}$; VIP, vasoactive intestinal peptide.

tic lamina remains unclear (Figure 2A). In the hypoxic animal model of pulmonary hypertension, the adventitial fibroblast appears to be the first cell activated in response to the hypoxic stimulus (5). What the initiating trigger or injury is that results in endothelial dysfunction with abnormal endothelial proliferation remains an area of intense investigation; possible players include: chronic hypoxia, inflammation, mechanical stretch, and/or shear stress, occurring in the setting of a genetic predisposition. Abnormalities in growth-suppressive genes, including TGFBR2 and the apoptosis-related gene $B A X$, have been reported (6). In plexiform lesions, which represent a distinct characteristic in idiopathic $\mathrm{PAH}$ as well as in other forms of PAH, TGFBR2 is rarely expressed. In the monocrotalineinduced rat model of pulmonary hypertension, the role of inflammation suggests that proinflammatory cytokines as well as chemokines are also intimately involved in the pathobiology of PAH (7). In addition, thrombotic lesions and platelet dysfunction appear to play a significant role (8). It is well appreciated that abnormalities in procoagulant activity as well as fibrinolytic function may be due to shear stress generating a thrombogenic surface with the subsequent development of thrombotic lesions. Increased plasma levels of fibrinopeptide A- and D-dimers support this hypothesis, with more recent studies suggesting that the interactions between platelets and pulmonary arterioles contribute to the progressive pulmonary vascular changes seen in PAH (8). This may result in a vicious cycle leading to various alterations in the production and activity of vasoactive and mitogenic mediators in addition to procoagulants released by platelets. These perturbations may also accelerate vasoconstriction by releasing thromboxane $\mathrm{A}_{2}$, platelet-activating factor, serotonin, PDGF, TGF- $\beta$, and VEGF. However, despite the multitude of perturbations that have been demonstrated in clinical PAH as well as in animal models of pulmonary hypertension, it remains unclear which are "causes" versus consequences of this disorder.

\section{PDGF inhibitor reverses pulmonary hypertension}

In this issue of the JCI, Schermuly et al. STI571 (imatinib mesylate) reverses pulmonary vascular remodeling in 2 different animal models of pulmonary hypertension (9) (Figure 2B). With PDGF known to be a potent mitogen and the progression of pulmonary hypertension associated with increased proliferation and migration of pulmonary VSMCs, inhibiting the effects of PDGF may prove efficacious in reversing pulmonary vascular disease. As the authors state, previous studies demonstrating PDGF upregulation in a lamb model with chronic intrauterine pulmonary hypertension further raises the possibility that inhibiting PDGF could be an efficacious therapeutic approach (10). As the PDGF receptors are members of the family of transmembrane receptor kinases, the autophosphorylation of which increases kinase activity, the authors investigated the effect of STI571, which targets the ATP-binding site of tyrosine kinases; STI571 is also an inhibitor report that the PDGF receptor antagonist of PDGF. Based on their hypothesis that altered PDGF signaling plays an important role in the pathobiology of PAH, the authors used the monocrotaline-induced pulmonary hypertension rat model as well as the chronic hypoxic pulmonary hypertension mouse model to demonstrate that administration of STI571, after the development of pulmonary hypertension, significantly reversed the pulmonary vascular changes. The authors also examined lung tissue from PAH patients that had undergone lung transplantation; the authors found significantly increased expression of PDGF in lung tissue from the PAH patients compared with control donor lung tissue. The authors concluded that a PDGF receptor inhibitor may be a novel therapeutic approach for the treatment of clinical PAH. STI571 is an approved anticancer drug that appears to be well tolerated $(11,12)$. Thus, the study by Schermuly et al. provides preclinical proof of concept for the clinical development of the PDGF receptor inhibitor STI571 as a novel targeted therapeutic agent for the treatment of pulmonary hypertension. A pilot proof-of-concept clinical trial evaluating the safety and efficacy of imatinib mesylate in PAH is in progress.

\section{Pathobiology of PAH}

As is seen in many of the other perturbations observed in the pathobiology of PAH, upregulation and activation of various signaling pathways becomes a self-perpetuating vicious cycle, which, regardless of the initiating trigger or stimulus, results in progressive pulmonary vascular obstructive disease. However, one must remain cognizant that with any attempt to interrupt one vicious cycle, other pathways that may also be involved could either further reverse or further accelerate the pulmonary vascular changes. It is an inherent problem that as we attempt to develop novel targeted therapeutic agents for PAH, examining 1 pathway in isolation can be misleading; as shown in Figure 1 , the postulated mechanisms involved in the pathobiology of PAH are complex, with potentially significant interactions.

There is also evidence to suggest that MMP expression is upregulated in PAH (13). In the report by Schermuly et al. (9), both MMP-2 and MMP-9 were significantly increased in the lung homogenates of the rats treated with monocrotaline when compared with control rats. Furthermore, this increase was reversed by STI571 administration. Most of the stimuli that cause acute pulmonary vasoconstriction also 
cause cell proliferation, e.g., endothelin, serotonin, and abnormalities in potassium channels. In addition, with mutations in bone morphogenetic protein (BMP) receptor 2 , activin receptor-like kinase 1 , endoglin, and TGF- $\beta$ receptors reported in patients with $\mathrm{PAH}$, the effects of STI571 on cell proliferation cannot be anything other than complex (14-17). The speculation that a failure of the growth inhibitory effects of BMPs contributes to the pulmonary vascular remodeling in $\mathrm{PAH}$ raises additional questions regarding the interaction of the TGF- $\beta$ /BMP pathways with other pathways known to be involved in the regulation of pulmonary vascular tone.

In addition to PDGF, various other growth factors, including basic FGF, IGF-1, and EGF have also been postulated to be involved in pulmonary vascular remodeling. Merklinger et al. recently reported that selective blockade of EGF receptor signaling may offer another novel approach to the treatment of PAH (18). Although reactive oxygen species have been suggested as inducers of PDGF expression and MMP signaling through EGF receptors, the induction of these growth factors may be more complicated. Similarly, hypoxia and shear stress have also been implicated, consistent with the reversal of pulmonary vascular changes observed by Schermuly et al. (9) in the hypoxic pulmonary hypertension mouse model.

As we proceed with further investigation with the goal of unraveling the complicat- ed interactive pathobiologic mechanisms initiating and perpetuating $\mathrm{PAH}$, we must also explore the potential roles of angiogenesis, apoptosis, and proteolysis. An example of an interaction of these various pathways, with respect to the monocrotaline-induced pulmonary hypertension rat model used in the Schermuly et al. study (9), is a study reporting that serine elastases activate MMPs and also suppress tissue inhibitors of MMPs $(19,20)$. In the monocrotaline-induced pulmonary hypertension rat model, an elevation in elastase activity has been seen as early as day 2 after injection of the toxin, whereas increased MMP activity is not increased until day 21. Thus, it appears that at least in the monocrotaline-induced pulmonary

A

Vascular remodeling in $\mathrm{PAH}$
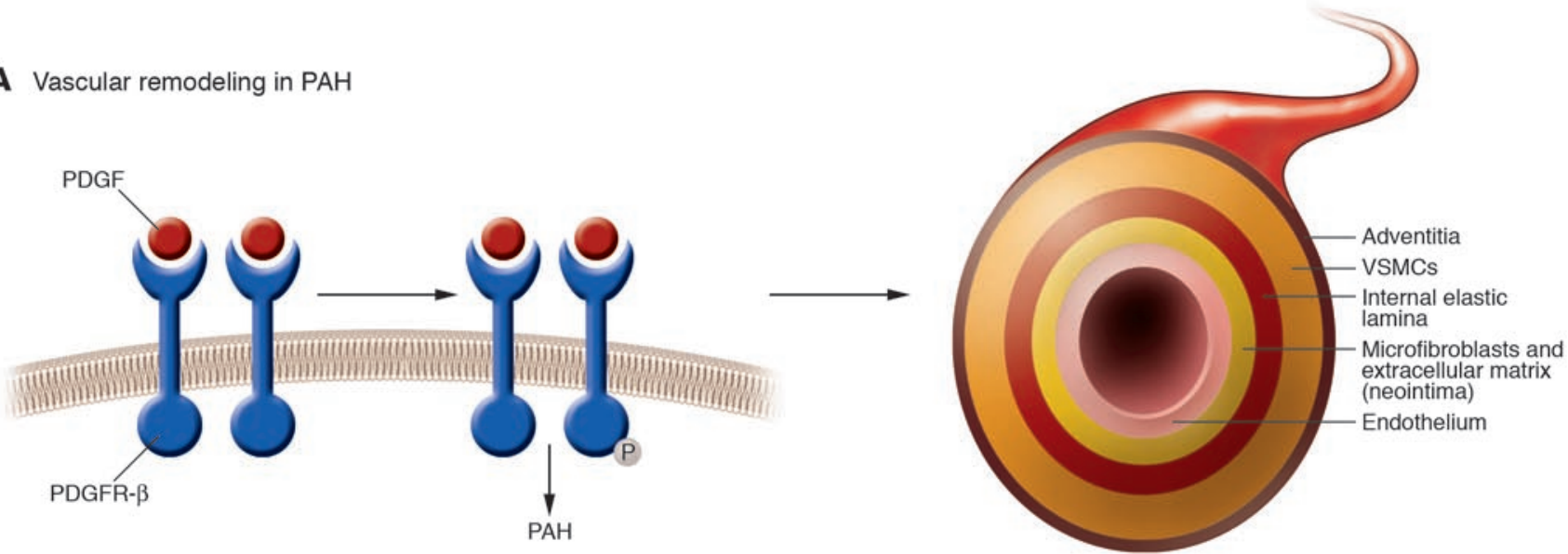

B STI571-induced reversal of pulmonary vascular remodeling
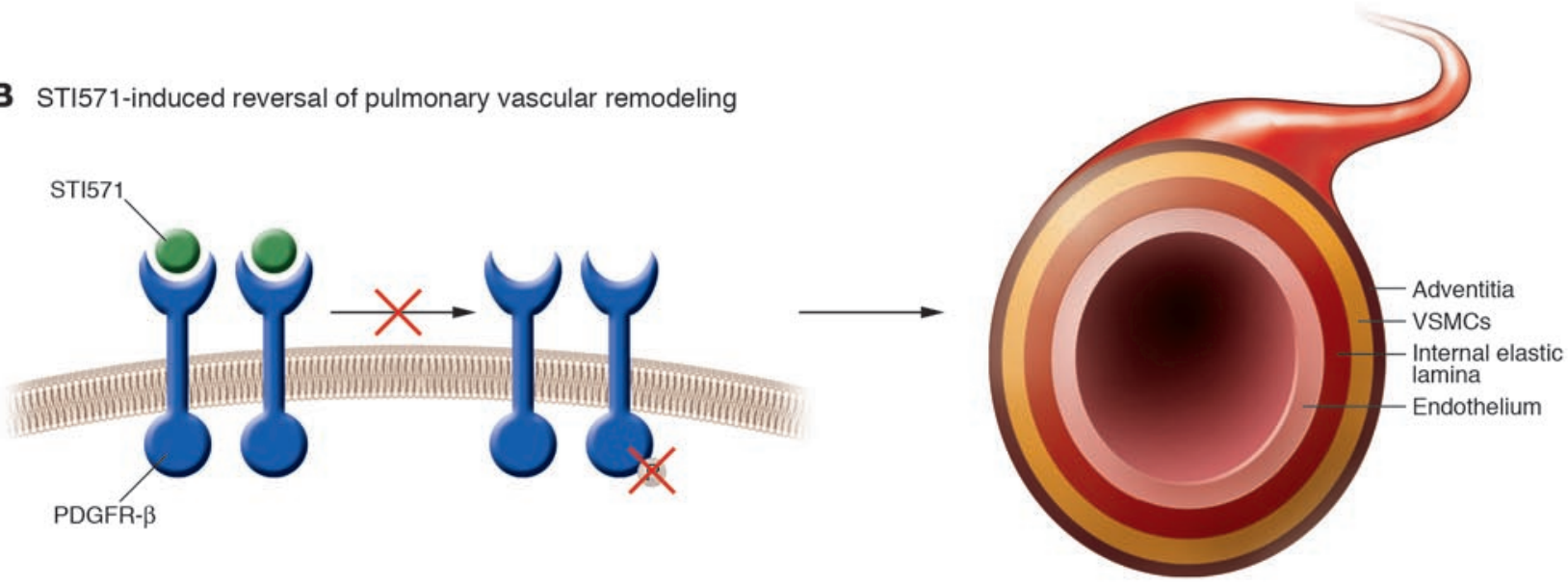

Figure 2

Schematic representation of pulmonary vascular remodeling in the small pulmonary arteries in PAH. (A) PDGF receptor (PDGFR) expression and phosphorylation $(\mathrm{P})$ in pulmonary arteries are increased in $\mathrm{PAH}$, which activates downstream signaling pathways that promote the abnormal proliferation and migration of VSMCs as well as the formation of a layer of microfibroblasts and extracellular matrix (termed the neointima) between the internal elastic lamina and the endothelium. These changes underlie the structural and functional abnormalities in the vessel wall that lead to pulmonary vascular disease. (B) In this issue of the $\mathrm{JCl}$, Schermuly et al. demonstrate that administration of the PDGF receptor antagonist STI571 induces a reversal of the pulmonary vascular remodeling in 2 different animal models of pulmonary hypertension. STI571 prevents phosphorylation of the PDGF receptor and consequently suppresses activation of downstream signaling pathways associated with PAH. 
hypertension rat model, elastase activity precedes MMP activity and subsequently induces MMP activity. The matrix degradation by MMPs and elastases further interacts with growth factors such as EGF, resulting in further induction of VSMC proliferation. These data raise the question of whether targeting this pathway using elastase inhibitors will be another effective therapeutic approach. Additional investigation is needed to determine which, if any, of these abnormalities initiate PAH and which ones are the best to target for therapeutic gain. It is clear that $\mathrm{PAH}$ has a multifactorial pathobiology. As we increase our understanding of the pathways involved and their interactions, we will hopefully increase the efficacy of novel therapeutic strategies for this severe disease and further improve the palliative therapy for PAH and ultimately cure PAH.

Address correspondence to: Robyn J. Barst, New York Presbyterian Pulmonary Hypertension Center, Columbia University College of Physicians \& Surgeons, 3959 Broadway, BHN 2-255, New York, New York 10032, USA. Phone: (212) 305-4436; Fax: (212) 3421443; E-mail: rjb3@columbia.edu.
1. Humbert, M., Sitbon, O., and Simonneau, G. 2004. Treatment of pulmonary arterial hypertension. N. Engl. J. Med. 351:1425-1436.

2. Barst, R.J., et al. 1996. A comparison of continuous intravenous epoprostenol (prostacyclin) with conventional therapy in primary pulmonary hypertension. The Primary Pulmonary Hypertension Study Group. N. Engl. J. Med. 334:296-301.

3. Rubin, L.J., et al. 2002. Bosentan therapy for pulmonary arterial hypertension. N. Engl. J. Med. 346:896-903

4. Galie, N., et al. 2005. Sildenafil citrate therapy for pulmonary arterial hypertension. N. Engl. J. Med. In press.

5. Stenmark, K.R., Gerasimovskaya, E., Nemenoff, R.A., and Das, M. 2002. Hypoxic activation of adventitial fibroblasts: role in vascular remodeling. Chest. 122:326S-334S

6. Yeager, M.E., Halley, G.R., Golpon, H.A., Voelkel, N.F., and Tuder, R.M. 2001. Microsatellite instability of endothelial cell growth and apoptosis genes within plexiform lesions in primary pulmonary hypertension. Circ. Res. 88:e2-e11.

7. Balabanian, K., et al. 2002. CX (3) C chemokine fractalkine in pulmonary arterial hypertension. Am. J. Respir. Crit. Care Med. 165:1419-1425.

8. Herve, P., et al. 2001. Pathobiology of pulmonary hypertension: the role of platelets and thrombosis. Clin. Chest Med. 22:451-458.

9. Schermuly, R.T., et al. 2005. Reversal of experimental pulmonary hypertension by PDGF inhibition. J. Clin. Invest. 115:2811-2821. doi:10.1172/JCI24838.

10. Balasubramaniam, V., et al. 2003. Role of plateletderived growth factor in vascular remodeling during pulmonary hypertension in the ovine fetus. $A m$. J. Physiol. Lung Cell Mol. Physiol. 284:L826-L833.

11. Cohen, M.H., et al. 2002. Approval summary for imatinib mesylate capsules in the treatment of chronic myelogenous leukemia. Clin. Cancer Res. 8:935-942.

12. Dagher, R., et al. 2002. Approval summary: imatinib mesylate capsules in the treatment of metastatic and/or unresectable malignant gastrointestinal stromal tumors. Clin. Cancer Res. 8:3034-3038.

13. Frisdal, E., et al. 2001. Gelatinase expression in pulmonary arteries during experimental pulmonary hypertension. Eur. Respir. J. 18:838-845.

14. Lane, K.B., et al. 2000. Heterozygous germline mutations in a TGF- $\beta$ receptor, BMPR2, are the cause of familial primary pulmonary hypertension. Nat. Genet. 26:81-84.

15. Deng, Z., et al. 2000. Familial primary pulmonary hypertension (gene PPH1) is caused by mutations in the bone morphogenetic protein receptor-II gene. Am. J. Hum. Genet. 67:737-744.

16. Trembath, R., et al. 2001. Clinical and molecular genetic features of pulmonary hypertension in patients with hereditary hemorrhagic telangiectasia. N. Eng. J. Med. 345:325-334.

17. Chaouat, A., et al. 2004. Endoglin germline mutation in a patient with hereditary hemorrhagic telangiectasia and dexfenfluramine-associated pulmonary arterial hypertension. Thorax. 59:446-448.

18. Merklinger, S.L., Jones, P.L., Martinez, E.C., and Rabinovitch, M. 2005. Epidermal growth factor receptor blockade mediates smooth muscle cell apoptosis and improves survival in rats with pulmonary hypertension. Circulation. 112:423-431.

19. Cowan, K.N., Jones, P.L., and Rabinovitch, M. 2000. Elastase and matrix metalloproteinase inhibitors induce regression, and tenascin- $C$ antisense prevents progression, of vascular disease. J. Clin. Invest. 105:21-34.

20. Cowan, K.N., et al. 2000. Complete reversal of fatal pulmonary hypertension in rats by a serine elastase inhibitor. Nat. Med. 6:698-702.

\title{
Give me A5 for lipoprotein hydrolysis!
}

\section{Martin Merkel ${ }^{1}$ and Joerg Heeren ${ }^{2}$}

1Department of Internal Medicine and 2IBM II: Molecular Cell Biology, University Hospital Hamburg-Eppendorf, Hamburg, Germany.

\begin{abstract}
APOA5 is a newly identified apolipoprotein that plays a crucial role in the regulation of plasma triglyceride levels. In several human studies, common APOA5 single nucleotide polymorphisms have been strongly associated with elevated plasma triglyceride levels. In this issue of the JCI, Marçais et al. report that the rare Q139X mutation in APOA5 leads to severe hypertriglyceridemia by exerting a dominant-negative effect on the plasma lipolytic system for triglyceride-rich lipoproteins (see the related article beginning on page 2862). The presented data support the idea that the molecular mechanism of APOA5 function may include the enhancement of binding between lipoproteins and proteoglycans at the vascular wall and activation of proteoglycan-bound lipoprotein lipase.
\end{abstract}

\section{A new cause of hypertriglyceridemia}

The function of lipoproteins in vascular wall biology continues to unfold in an

Nonstandard abbreviations used: APOA5, human apolipoprotein A-V; Apoa5, murine apolipoprotein A-V; LPL, lipoprotein lipase.

Conflict of interest: The authors have declared that no conflict of interest exists.

Citation for this article: J. Clin. Invest. 115:2694-2696 (2005). doi:10.1172/JCI26712. increasingly complex story. It was more than 20 years ago that the majority of apolipoproteins known to play a major role in lipid metabolism were identified, and 9 of them (APOA1, APOA2, APOA4, APOB48, APOB100, APOC1, APOC2, APOC3, and APOE) have long been known to be most relevant to the regulation of lipoproteins. In contrast, additional apolipoproteins have only recently been identified, and their roles in lipoprotein metabolism are still being uncovered. For instance, APOM and its important function in HDL formation were just described this year (1). An important discovery in triglyceride biology was made in 2001 when a new member of the apolipoprotein family, human apolipoprotein A$\mathrm{V}$ (APOA5), was identified by postgenomic techniques. It was described independently by 2 groups; the first group used comparative sequencing (2), and the second group identified rat Apoa5 after observing the marked upregulation of the Apoa5 gene during liver regeneration (3). Since its plasma concentration is only about one-thousandth that of some other apolipoproteins (e.g., APOA1 and APOA2) (4), the relevance and function of APOA5 was not immediately fully recognized.

In this issue of the JCI, Marçais et al. describe a truncation mutation of APOA5 associated with severe familial hypertriglyc- 